\title{
Measurement of Opening Displacement of Single and Bifurcated Notches by Moiré Interferometry
}

\author{
S. Suzuki ${ }^{1}$, T. Miyashita ${ }^{2}$, H. Kimura ${ }^{3}$, and S. Nishikita ${ }^{1}$ \\ ${ }^{1}$ Toyohashi University of Technology, Tempaku-cho, Toyohashi, Aichi, 441-8580, Japan \\ ${ }^{2}$ YEC, Nishikaizuka, Iwata, Shizuoka, 438-0026, Japan \\ ${ }^{3}$ JFE Steel Co., Koukan-cho, Fukuyama, Hiroshima, 721-0931, Japan
}

\begin{abstract}
Static experiments are performed to confirm the COD method that is the only method to measure the energy release rate of fast propagating cracks just after bifurcation. The crack opening displacement (COD) of the mother cracks of bifurcated cracks is measured in static condition by Moiré interferometry. Thin notches are used instead of cracks. The bifurcation angle is 13.5 degrees that is the same as the angle of bifurcation of fast propagating cracks in PMMA. The measured CODs are proportional to the square root of the distance from the nominal tip of the mother notch. Stress intensity factor of the bifurcated notch is obtained from the measured CODs through the formula of the COD of single cracks. The experimental results say that the stress intensity factor of a bifurcated crack has the same value as that of the single crack whose length is the same as that of the bifurcated crack. This is caused by the small bifurcation angle of 13.5 degrees. It is concluded that the COD method is correct to measure the energy release rate of rapidly bifurcating cracks.
\end{abstract}

\section{Introduction}

When brittle materials break under external force, there appear fast propagating cracks that are of opening mode and whose speed is from 200 to $2000 \mathrm{~m} / \mathrm{s}$. When the crack speed is high enough, a fast propagating crack bifurcates into two cracks suddenly. Bifurcation is a characteristic feature of fast propagating cracks, however, the mechanism of the rapid crack bifurcation has not been fully understood.

One of the important problems to figure out the bifurcation mechanism is the continuity of energy release rate at bifurcation. After bifurcation, the area of the crack surfaces that are newly made by the crack extension of unit length is twice as large as that before bifurcation. Consequently, if the crack speed after bifurcation is the same as that before bifurcation, the energy release rate of the crack after bifurcation must increase discontinuously to twice as large as that before bifurcation.

The (dis)continuity of energy release rate at bifurcation had been an open problem, because there was no method to measure the energy release rate after bifurcation. But recently, Suzuki et al.[1, 2] measured the energy release rate of fast propagating cracks after bifurcation, and showed that the energy release rate is continuous across the bifurcation point. Their basic idea to measure the energy release rate is as follows. 
(1) The crack opening displacement, COD, of a single crack is proportional to the square root of distance from the crack tip (Fig.1(a)), and the energy release rate can be obtained by measuring the COD through the formulae of dynamic fracture mechanics.

(2) A bifurcated crack becomes a single crack when the bifurcation angle $\theta$ tends to zero (Fig.1(b)). Hence, if the bifurcation angle is small, it is expected that the COD of the mother crack is approximately proportional to $\sqrt{r}$.

(3) Under the above condition, the energy release rate after bifurcation can be obtained from the COD of the mother crack through the formulae for the single crack.

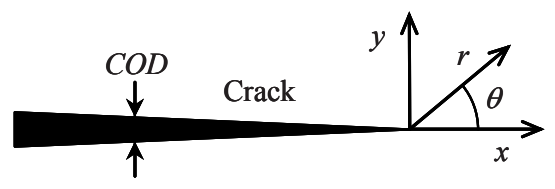

(a)

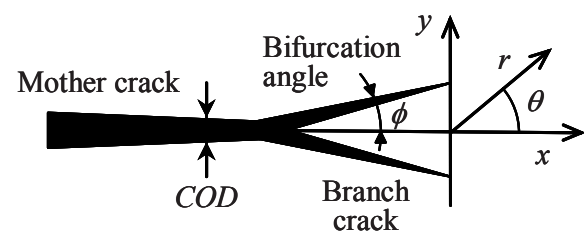

(b)

Fig. 1 (a) Single crack and COD. (b) Symmetrically bifurcated crack.

The present study performs static experiments to proof the validity of the above COD method to measure the energy release rate of bifurcated cracks $[3,4]$. Single and bifurcated notches are used instead of cracks, and CODs are measured by Moiré interferometry [5].

\section{Experimental method}

\subsection{Specimen}

Figure 2(a) illustrates the specimen used in the present study. The specimen is $250 \mathrm{~mm}$ long, $250 \mathrm{~mm}$ wide and $3 \mathrm{~mm}$ thick, and is made of PMMA. There is a notch from the left boundary toward the center of the specimen. A pair of tensile forces is applied at the holes near the upper and lower boundaries of the specimen. The tinsile forces are $108 \mathrm{~N}, 206 \mathrm{~N}, 304 \mathrm{~N}$ and $402 \mathrm{~N}$.

There are two kinds of notches. One is a single notch (Fig.2(b)), and the other is bifurcated notch (Fig. 2(c)). The entire length of the notches is $125 \mathrm{~mm}$ that is kept constant. The width of the notches is $0.3 \mathrm{~mm}$. In the case of bifurcated notches, the lengths of branch notches are $4.5 \mathrm{~mm}$, $6.0 \mathrm{~mm}$ and $12.0 \mathrm{~mm}$, and the bifurcation angle $\phi$ is 13.5 degrees that is the same as the average value of the bifurcation angles of fast propagating cracks in PMMA.

A thin diffraction grating is pasted around the notch tip to measure the opening displacement of the notch by Moiré interferometry. The spatial frequency of the grating is 4881 ines $/ \mathrm{mm}$.

\subsection{Moiré interferometry for COD measurement}

Figure 3 illustrates the optical system for Moiré interferometry used in the present study. The light beam emitted from the He-Ne laser passes through lens L1 and L2, and becomes the parallel light beam. The parallel beam is divided into two beams by beam splitter BS. The light beam reflected by the beam splitter becomes collimated beam A, and the light beam transmitted through 
(a)

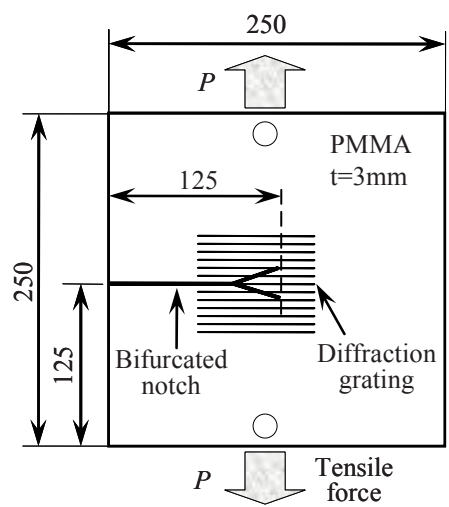

(b)

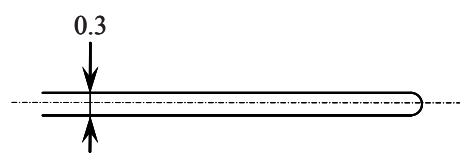

(c)

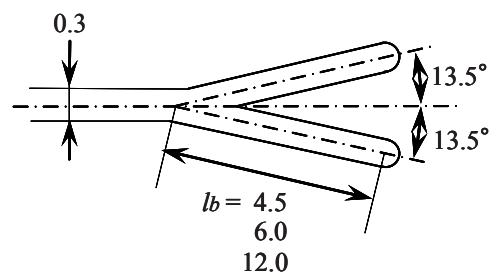

Fig.2 (a) Specimen with notch. (b) Single notch. (c)Bifurcated notch.

the beam splitter becomes collimated beam B. The two collimated beams fall onto the grating pasted on the specimen surface, and are diffracted by the grating. The first order diffraction beam of collimated beam A and the minus first order diffraction beam of collimated beam B make the interference fringes on the specimen surface that represent the displacement of the specimen. The two diffracted beams propagate perpendicularly to the specimen surface, and enter into the camera, which takes the photographs of interference fringes on the specimen.

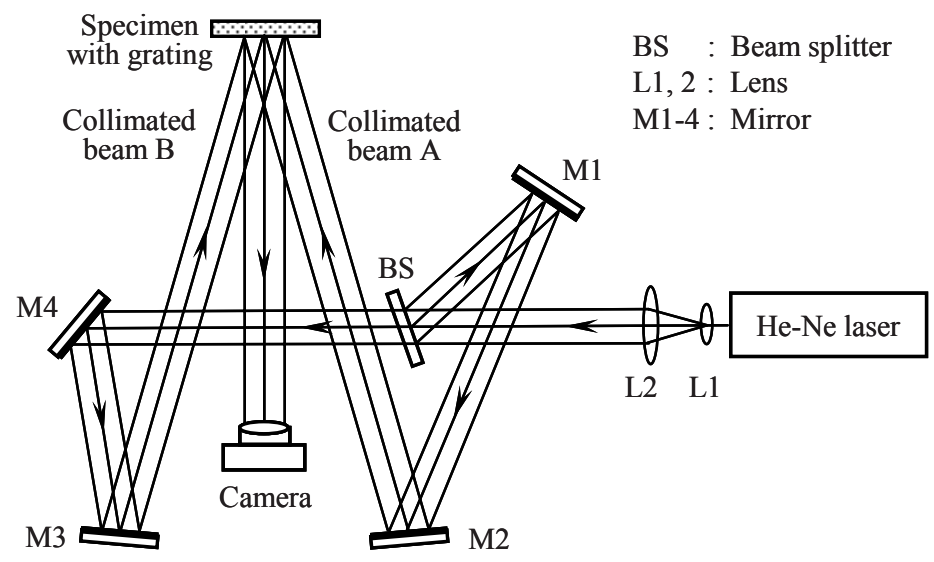

Fig. 3 Optical system for Moiré interferometry.

\section{Results}

\subsection{Moiré interference fringes}

Figure 4 shows an example of Moire interference fringes around the notches. Figure 4(a) is a single notch, and (b) is the bifurcated notch whose length of the branch notches is $6 \mathrm{~mm}$. The applied 
tensile force is $304 \mathrm{~N}$. The interference fringes are the equi-displacement lines in the direction perpendicular to the notch. The displacement between each fringe and the next is $1.02 \mu \mathrm{m}$. The difference of the fringe order is measured along the notches on the photographs to obtain CODs. Similar interference fringes are observed with other specimens of different notch length and under different tensile forces.
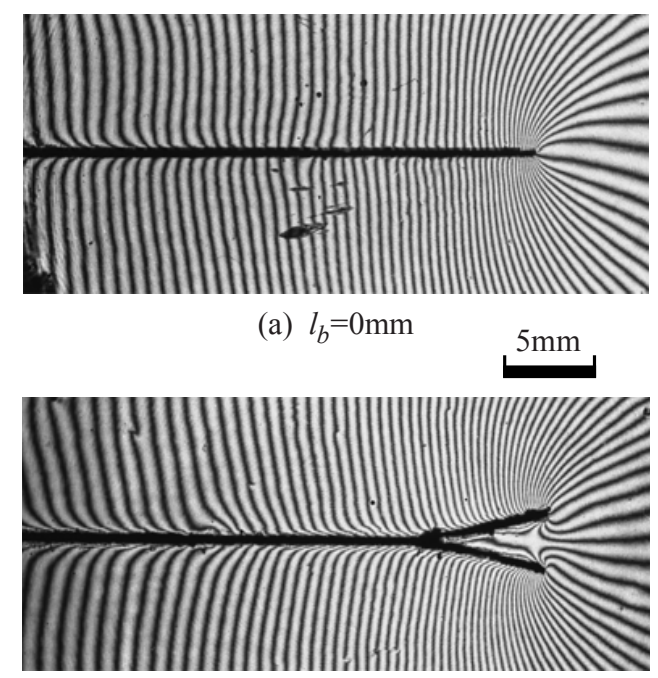

(b) $l_{b}=6.0 \mathrm{~mm}$

Fig. 4 Interference fringes around (a) a single notch, and (b) bifurcated notch.

The tensile force applied to the specimen is $304 \mathrm{~N}$.

\subsection{COD measurement}

Figure 5 represents the CODs measured from the interference fringes shown in Fig. 4. The applied tensile force is changed from 108 to $402 \mathrm{~N}$. Figure 5(a) is the COD of the single notch shown in Fig. 4(a), and Fig. 5(b) is the CODs of mother notch of the bifurcated notch shown in Fig. 4(b). The horizontal axes are the distance $r$ from the notch tip, and the vertical axes are CODs. Both the axes are in log scale. The CODs were measured up to $r=25 \mathrm{~mm}$.

The straight lines in the figure are obtained by assuming that $C O D \propto r^{\lambda}$. The exponent $\lambda$ was determined with the measurement data of COD by the least square method. The graphs tell that not only the COD of the single notch but also the CODs of the mother notch of the bifurcated notch are proportional to $r^{\lambda}$ within the region of $r$ less than $25 \mathrm{~mm}$.

\subsection{Stress intensity factor}

Figure 6 shows the stress intensity factor $K_{I}$ obtained from the measured COD data shown in Fig. 5. The horizontal axis is the distance $r$ from the notch tip. Figure 6(a) is the stress intensity factor of the single notch. The values of $K_{I}$ are kept constant even if the distance $r$ from the notch tip varies. This means that the measurement was carried out within the singular stress field that exists in the vicinity of the notch tip. 


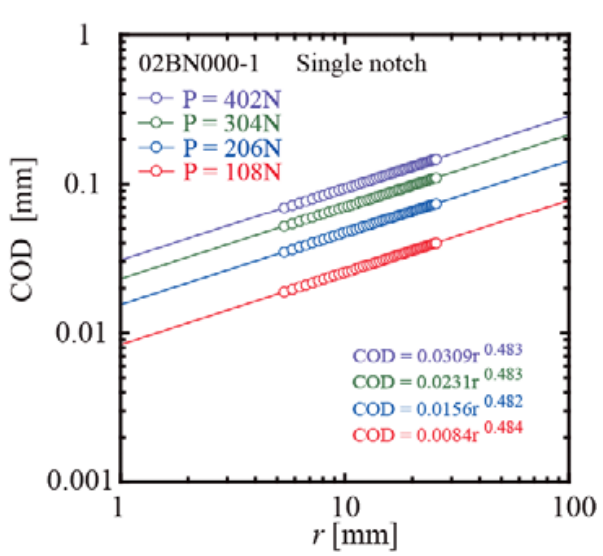

(a)

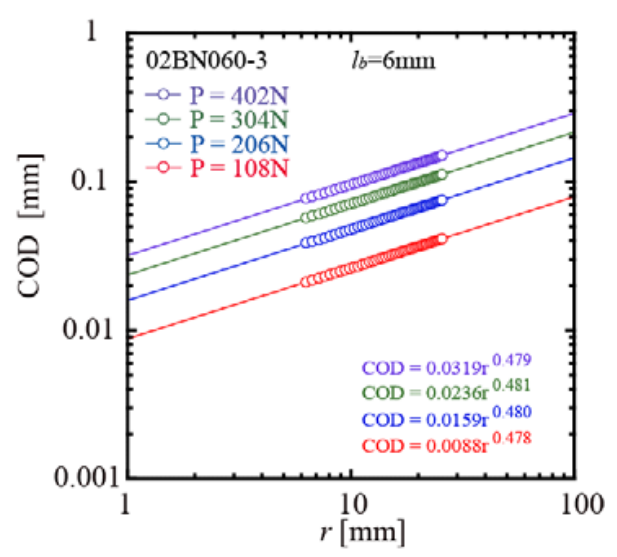

(b)

Fig.5 Interference fringes around (a) a single notch, and (b)-(d) bifurcated notches.

The tensile force applied to the specimen is $304 \mathrm{~N}$.

Figure 6(b) represents the stress intensity factor obtained from the measured COD of mother notch shown in Fig. 5(b). The stress intensity factor obtained from the COD of mother notch is also kept constant even if the distance $r$ from the nominal tip of the mother notch changes. It can thus be said that the stress intensity factors of the bifurcated notch is correctly obtained from the COD of the mother notch.

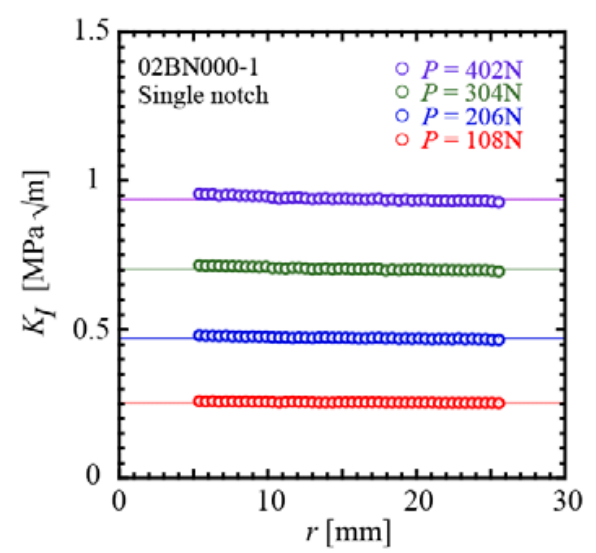

(a)

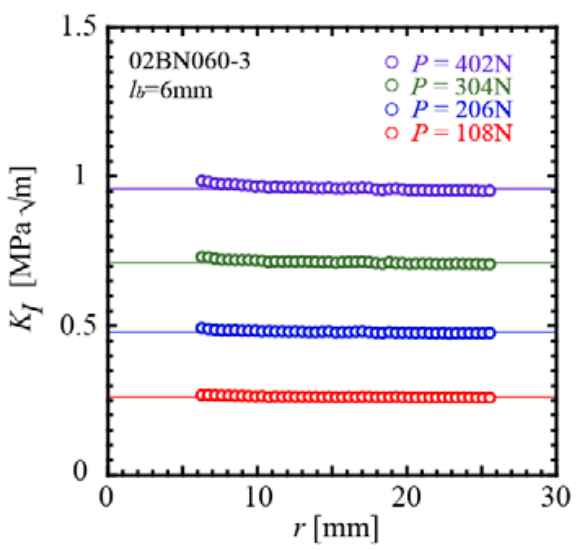

(b)

Fig. 6 Stress intensity factors of a single notch and a bifurcated notch.

Figure 7 shows the stress intensity factors $K_{I B}$ of the bifurcated notches normalized with the stress intensity factor $K_{I S}$ of the single notch under the same tensile force. The horizontal axis is the length $l_{b}$ of the branch notch. The graph says that the stress intensity factors of bifurcated notches 
are the same as that of the single notch, even though the length of branch notch changes. This fact means that the stress intensity factors of bifurcated notches can be obtained by measuring the COD of the mother notches under the condition that the bifurcation angle is 13.5 degrees.

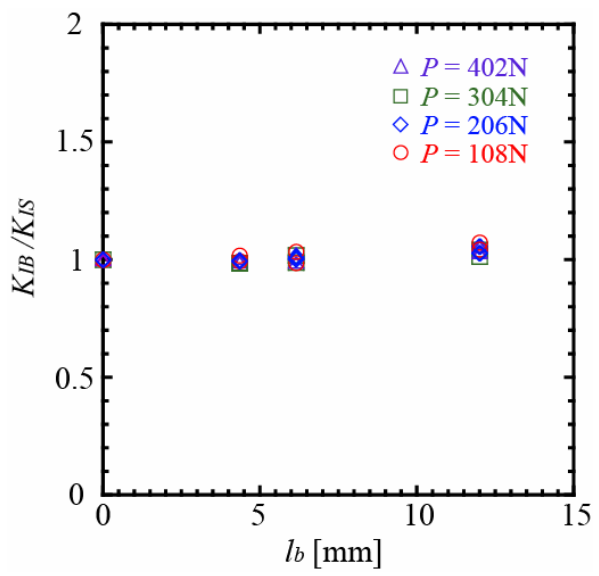

Fig. 7 Stress intensity factor of branch notches normalized by that of the single notch as a function of the length of branch notch.

\section{Conclusions}

(1) The crack opening displacement, COD, of the mother crack is proportional to the square root of the distance $r$ from the crack tip, if the bifurcation angle is small and if the length of the branch cracks is much smaller than the entire length of the crack.

(2) The bifurcation angle of 13.5 degrees of fast propagating cracks is small enough to obtain the stress intensity factor of bifurcated cracks.

(3) The COD method is appropriate to measure energy release rate just after dynamic crack bifurcation

\section{References}

1. S. Suzuki, K. Sakaue and K. Iwanaga, J. Mech. Phys. Solids, 55 (2007), 1487-1512.

2. S. Suzuki and K. Sakaue, JSME Int. J., Series A, 47 (2004), 264-273.

3. S. Suzuki and T. Miyashita, J. Solid Mech. and Material Eng., 2 (2008), 25-37.

4. S. Suzuki, T. Miyashita, H. KIMURA and S. NISHIKITA, J. Solid Mech. and Material Eng., 3 (2009), 782-795.

5. D. Post, Moiré Interferometry, Handbook on Experimental Mechanics, (1987), pp.314-387, Prentice-Hall. 